\title{
Intégration du marché européen de la Défense et politiques d'offsets
}

Une analyse en termes de coûts de changement et d'externalités technologiques

Adel Ben Youssef et Gueorgui lanakiev

\section{(2) OpenEdition}

Journals

Édition électronique

URL : http://journals.openedition.org/ei/284

DOI : 10.4000/ei.284

ISSN : 2553-1891

Éditeur

Association Économie et Institutions

Édition imprimée

Date de publication : 30 octobre 2009

Pagination : 113-138

ISSN : 1775-2329

Référence électronique

Adel Ben Youssef et Gueorgui lanakiev, «Intégration du marché européen de la Défense et politiques d'offsets », Économie et institutions [En ligne], 12-13 | 2009, mis en ligne le 31 janvier 2013, consulté le 30 avril 2019. URL : http://journals.openedition.org/ei/284 ; DOI : 10.4000/ei.284 


\title{
Intégration du marché européen de la défense et politiques d'offsets : une analyse en termes de coûts de changement et d'externalités technologiques ${ }^{1}$
}

\author{
Adel Ben Youssef ${ }^{2}$ et Gueorgui Ianakiev ${ }^{3}$
}

\section{Introduction}

L'intégration économique européenne en matière de produits et services de la défense demeure encore faible. En dépit d'un contexte international marqué par un recours croissant aux interventions militaires et une convergence des analyses sur la nécessité de mettre sur pieds une force armée européenne commune crédible, l'Europe de la défense reste à construire.

Le marché européen demeure extrêmement fragmenté, constitué en réalité d'une multitude de marchés nationaux plus ou moins indépendants, mais de taille réduite. Dans une industrie où les coûts fixes, en particulier ceux de recherche et développement, sont particulièrement importants, l'efficacité de l'industrie européenne en est affectée. Elle doit pourtant faire face à une concurrence internationale importante, en particulier en provenance des entreprises américaines adossées à un marché national relativement protégé et dont la taille est significativement supérieure à celle des marchés de tous pays membres de l'Union Européenne pris ensemble. Etant donnée l'importance du secteur, aussi bien sur le plan stratégique que sur le plan technologique, construire un marché intégré des produits de défense au sein de l'UE constitue un enjeu crucial. Lorsqu'une telle optique est envisagée, une question se pose, donnant lieu à des débats acharnés traduisant des positions souvent radicalement opposées : celle du rôle des politiques d'offset.

Les offsets constituent des dispositions qui, ajoutées à un contrat d'importation de biens et/ou services par une entité publique, incitent les entreprises exportatrices étrangères à effectuer des activités répondant à des objectifs de l'entité importatrice autres que l'acquisition des éléments faisant l'objet du contrat initial. L'effet incitatif est véhiculé par le conditionnement de l'obtention du contrat principal par la fourniture des contreparties de compensation. D'un

1 Nous tenons à remercier vivement Thomas Le Texier et David Versailles pour une relecture d'une première version de ce travail. Les erreurs et les omissions demeurent de notre seule responsabilité.

2 GREDEG, Université de Nice Sophia Antipolis

3 ADIS - Université Paris Sud et Commission européenne

113 Economie et Institutions - $\mathrm{n}^{\circ} 12 \& 13-2008-2009$ 
point de vue théorique, ces dernières possèdent la propriété d'additionnalité, qui traduit la mesure dans laquelle, suite aux obligations de compensation, l'exportateur initial effectue, auprès de l'acheteur, des achats, des dépenses ou toutes autres opérations supérieures à celles qui auraient été effectuées en l'absence de l'accord d'offset.

L'analyse de ce phénomène est d'une importance cruciale dans la perspective de la création d'un marché européen des produits de défense. Les offsets sont souvent dépeints comme des mesures protectionnistes inefficaces et aux effets négatifs sur la division internationale du travail. Le recours aux offsets peut cependant être expliqué par l'existence simultanée de mesures nationales protectionnistes et de barrières naturelles, telles que des imperfections informationnelles et des coûts de changement de fournisseur, limitant l'établissement spontané de partenariats industriels transfrontaliers. Dans un tel cadre ils peuvent également exercer une action positive en tant que vecteur d'intégration du marché des produits de défense.

L'objet de cet article est double, d'une part nous chercherons à caractériser, en mobilisant les outils de l'économie industrielle, les motivations qui expliqueraient la spécificité de ce type de commerce. Nous chercherons à décrire les politiques de compensation internationale selon l'approche néo-institutionnaliste et de comprendre leurs logiques contractuelles. D'autre part, nous tenterons de comprendre les conséquences relatives à la prise en compte de ce phénomène sur la dynamique d'intégration du marché européen des produits de défense. En d'autres termes, en quoi les effets des barrières mentionnées peuvent se renforcer mutuellement et comment la simple suppression des barrières réglementaires pourrait s'avérer insuffisante.

Pour ce faire, dans une première section nous caractériserons l'ampleur du phénomène au niveau international et plus particulièrement dans l'espace économique européen. Dans une seconde partie, nous élaborerons une grille d'analyse théorique de ce phénomène dans une approche fondée sur l'existence de coûts de transactions et d'externalités technologiques. Enfin, dans une troisième partie, fort de la grille de lecture élaborée, nous détaillerons les conséquences de leur prise en compte sur l'établissement du marché européen de la défense.

\section{Section 1: L'importance des offsets dans le commerce international}

Le recours aux offsets n'a cessé de s'étendre depuis les années 1970, jusqu'au point de constituer, aujourd'hui, le principal trait caractéristique du commerce d'équipements de défense. La

114 Economie et Institutions - $\mathrm{n}^{\circ} 12 \& 13-2008-2009$ 
quasi-totalité des pays importateurs d'armement possède désormais une politique d'offset, que celle-ci soit formelle ou informelle. Le pourcentage d'offset, défini comme le rapport de la valeur des compensations demandées sur la valeur du contrat initial d'achat d'équipements de défense, se situe fréquemment au-dessus de 100\% et peut parfois même atteindre les 300\%.

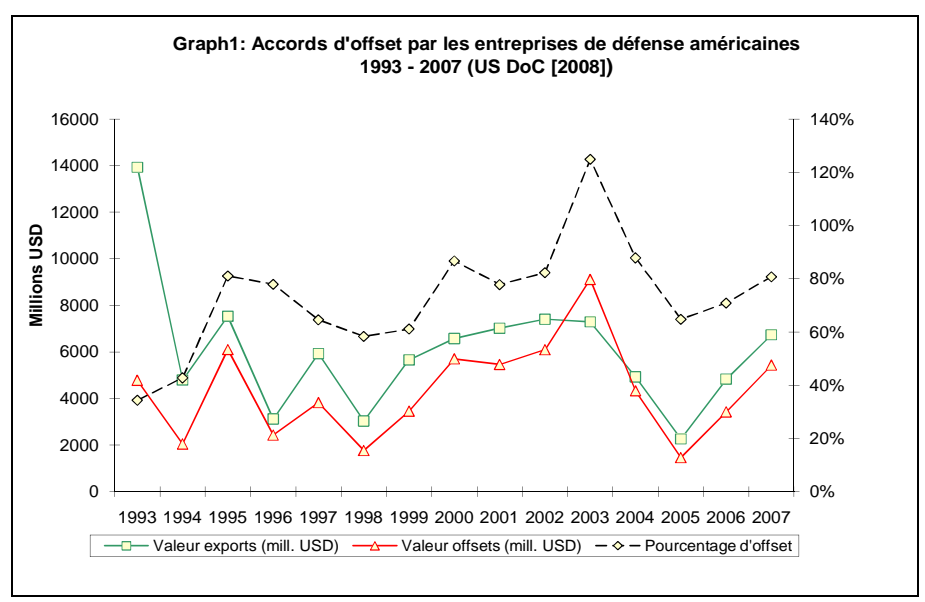

Le Graphique 1, basé sur les données collectées par le Département du Commerce des Etats-Unis ${ }^{4}$, permet d'illustrer la tendance. Sur la période 1993-2007 les entreprises américaines ont conclu 625 accords d'offset pour une valeur globale de plus de 65 milliards de dollars représentant $80 \%$ de la valeur des contrats initiaux d'exportation. Le pourcentage d'offset en fin de période était d'environ $72 \%$, ce qui constitue le double de la valeur constatée en début de période. Le Graphique 1 montre que le pourcentage d'offset suit une tendance globalement croissante, malgré un fléchissement suivant le pic historique de $125 \%$ atteint en 2003. Sur la même période plus 9000 transactions consécutives à des obligations d'offset ont été réalisées dans 48 pays différents et pour une valeur d'environ 45 milliards de dollars.

Il est important de souligner que les politiques d'offset des pays européens sont particulièrement exigeantes. Une étude commandée par l'Agence Européenne de la Défense ${ }^{5}$ (AED) montre

4 United States Department of Commerce, Offsets in Defense Trade, editions diverses (dernière décembre 2008).

5 FOI et SCS [2007], Study on the Effects of Offsets on the Development of a European Defence Industry and Market. L'étude a été financée par l'AED et

115 Economie et Institutions - n¹2\&13-2008-2009 
que le pourcentage d'offset demandé par les pays de l'Union Européenne est en moyenne de $135 \%$ sur la période 2000-2006. D’après les rapports du Département du Commerce américain les pourcentages liés aux accords avec l'Autriche et la Pologne sont d'environ $170 \%$ et le pourcentage moyen requis en Europe est sensiblement supérieur à celui du reste du monde. Ainsi, les accords conclus par les entreprises américaines avec des pays européens représentaient plus de $65 \%$ de la valeur des accords d'offset, mais seulement $48 \%$ de celle des contrats de vente reliés.

\section{a) Le contexte international du commerce des produits de la défense}

Il n'est pas possible de rendre compte de ce rôle croissant des offsets sans prendre en compte les caractéristiques fondamentales du secteur de la défense. Ceux-là constituent autant de facteurs permettent de comprendre l'intérêt soutenu pour les politiques d'offset. On peut, en particulier, citer la hausse structurelle des coûts des équipements militaires conjuguée aux difficultés budgétaires consécutives à la fin de l'affrontement Est-Ouest, le protectionnisme traditionnel toujours présent en matière d'armement et la structure industrielle du secteur avec un rôle crucial joué par les grands intégrateurs de systèmes.

La hausse structurelle des coûts des équipements militaires constitue probablement le phénomène le plus frappant, caractéristique de l'industrie de défense. Une augmentation annuelle des coûts unitaires de production de $5 \%$ à $10 \%$ en termes réels (Kirkpatrick [1995, 2004] et Pugh [1993]) ne peut être compensée par une augmentation équivalente des dépenses militaires. Or, ce phénomène semble être à l'œuvre sur l'ensemble de la période suivant la Seconde Guerre Mondiale. Les effets sur le plan industriel en sont conséquents, en particulier pour les petits et moyens pays pour qui le maintien d'une industrie de défense indépendante devient illusoire. Il en résulte une nécessité de spécialisation de niche conjuguée à un accès aux marchés d'exportation.

A cette tendance de long terme est venu s'ajouter le choc conjoncturel consécutif à la fin de la Guerre Froide et à l'effondrement de l'URSS. Celui-ci a induit une forte contraction des dépenses militaires. Dans les pays membres de l'OTAN, à la fin des années 1990 les dépenses militaires représentaient environ 70-75\% de leurs niveaux de la fin de la décennie précédente. De plus, dans un contexte où la menace stratégique semblait s'estomper, la

est disponible sur le site de l'Agence. Elle couvre 24 pays membres, à l'exception du Danemark, de la Bulgarie et de la Roumanie.

116 Economie et Institutions - $\mathrm{n}^{\circ} 12 \& 13-2008-2009$ 
justification même des dépenses militaires a été rendue sensiblement plus difficile.

Un autre facteur important concerne la persistance d'un fort niveau de protection des marchés nationaux des produits de défense, en particulier dans les grands pays producteurs. Le secteur s'est traditionnellement développé dans un cadre national protégé justifié par la volonté d'indépendance stratégique. Aujourd'hui, le protectionnisme est toujours présent, y compris à l'intérieur de l'Union Européenne, contribuant à un morcèlement fort et à d'importantes duplications. En 2005, les Etats Membres de 1'UE consacraient uniquement $18 \%$ de leurs dépenses d'équipement militaire à des programmes en coopération. En conséquence, il existait dans l'UE 89 programmes d'armement différents contre 27 aux Etats-Unis, dont les dépenses d'équipement sont pourtant trois fois supérieures à celles combinées des pays membres de l'UE (Commission Européenne [2007b]).

Enfin, il convient de noter la structure particulière de l'industrie de la défense. Les équipements de défense sont des produits complexes, souvent décrits comme des "systèmes de systèmes ". Le développement et la production de nombreux éléments constitutifs sont réalisés par des entreprises spécialisées agissant sous la coordination d'un intégrateur de systèmes. Le rôle de ce dernier est crucial car son action a pour effet de structurer le tissu industriel. Dans ce cadre, pour les entreprises réalisant des sous-systèmes et des composants l'accès au marché est synonyme à l'accès aux chaînes d'approvisionnement des grands intégrateurs de systèmes.

La combinaison de ces facteurs permet de mieux comprendre l'attrait des offsets. Ces-derniers constituent un moyen de favoriser l'accès de l'industrie des pays importateurs aux marchés étrangers. Cet accès est crucial étant donné le rétrécissement des marchés nationaux consécutif à la hausse des coûts. Dans le cadre de leurs commandes, les Etats importateurs traitent directement avec les grands intégrateurs de systèmes dont les chaînes d'approvisionnement constituent le marché cible auquel l'accès devrait être garanti. De même, les offsets peuvent faciliter la justification politique des contrats d'importation d'armement en permettant aux gouvernements importateurs de mettre en avant les retombées économiques des compensations qui seront reçues.

117 Economie et Institutions - nº $12 \& 13-2008-2009$ 


\section{b) Les transactions emblématiques en matière d'offsets dans l'espace économique européen}

Certaines transactions emblématiques ont aussi contribué à rendre les politiques d'offset populaires. En premier lieu, la vente de 348 avions de combat F-16 à un groupe de 4 pays européens comportant la Belgique, les Pays-Bas, la Norvège et le Danemark. Souvent appelé "le contrat du siècle", cette transaction de 2.8 milliards de dollars fut conclue en 1975. Elle était assortie d'un programme d'offset sans précédent qui avait joué un rôle important dans le choix de l'appareil américain au détriment de son concurrent français le Mirage F1. Les quatre pays importateurs ont pu bénéficier d'un programme de participation industrielle à la production de F-16 allant largement au-delà de leur simple commande. La part des pays importateurs dans la production des 348 appareils commandés s'élevait à $40 \%$ de la valeur de la commande et incluait l'assemblage final des appareils et des réacteurs. Ils obtenaient également une part équivalente à $15 \%$ de la valeur des ventes à des pays tiers et $10 \%$ de la valeur des F-16 achetés par l'US Air Force. Un tel programme d'offset fournit aux pays importateurs une participation permanente à la production du système de défense concerné, positionnant les entreprises nationales comme partenaires durables de l'intégrateur de systèmes américain. Dans le cadre de la fabrication de F-16, cette participation se poursuit toujours plus de 30 ans plus tard car l'appareil fait toujours l'objet de commandes de la part de pays tiers.

Une seconde transaction eut lieu au milieu des années 1970, qui allait aussi jouer un rôle important : l'achat d'avions de combat F-5 par la Suisse. Les offsets requis par le gouvernement Suisse avaient pour objectif de permettre l'accès de l'industrie nationale au marché américain, tout en garantissant les transferts technologiques nécessaires au bénéfice de l'industrie nationale, devant permettre à celle-ci d'acquérir les capacités nécessaires pour assurer à la Suisse un haut degré d'indépendance stratégique. A la différence des offsets liés à la vente de F-16 au groupe des quatre pays européens, l'importance de la participation aux commandes effectuées par des pays tiers demeurait ici très limitée à cause du caractère restrictif de la politique d'exportation d'armement suisse. Northrop et General Electric, principaux producteurs du F-5, établirent des relations avec des entreprises suisses qui survécurent à l'extinction du programme d'offset en raison de la découverte de partenaires fiables et performants dans de nombreux secteurs de l'économie suisse. Parmi les exemples les plus frappants fut l'achat par General Electric de fraiseuses suisses à propos desquelles les responsables de l'entreprise américaine déclarèrent qu'ils " n'avaient jamais vu un tel

118 Economie et Institutions - n²12\&13-2008-2009 
niveau de qualité...ceci a conduit à une diminution de la proportion des coûts d'adaptation de $20 \%$ à $2 \%$ " (Udis [1996] ${ }^{6}$ ).

\section{c) Un phénomène peu étudié dans la littérature économique et des statistiques peu disponibles}

En dépit du rôle croissant joué par les offsets, ceux-ci restent peu connus en dehors des milieux professionnels concernés. La littérature économique sur le sujet demeure largement descriptive, avec des développements théoriques relativement limités. En particulier, elle n'arrive pas à fournir une explication robuste au recours aux offsets en tant qu'instrument visant l'établissement de partenariats industriels transfrontaliers durables. Pourtant, de nombreux exemples existent, montrant que les offsets peuvent mener à la découverte de fournisseurs efficaces dans les pays initialement importateurs.

D'après l'étude de Martin \& Hartley [1995a] sur les offsets accordés par les entreprises britanniques, dans le cadre de 6 ventes sur un total de 11 , les compensations ont mené à la découverte de nouvelles sources d'approvisionnement moins chères. L'intention était de poursuivre ces relations après l'extinction des obligations d'offset. Une étude de cas particulièrement intéressante, concernant l'Israel, peut aussi être trouvée chez Redlich \& Miscavage [1996]7. D'après les auteurs, consultants en matière d'offsets, les représentants des entreprises américaines souhaitant vendre des avions de combat à l'Etat d'Israel ont reconnu que la nécessité de développer un programme d'offset a joué un rôle da catalyseur et a permis la mise en évidence d'opportunités qui n'auraient pas été détectées autrement. Sur 100 projets potentiels, 30 ont été considérés comme hautement prioritaires et plusieurs contrats résultant ont été signés avant même que le vainqueur de l'appel d'offre sur le contrat initial concernant les avions de combat ait été annoncé

Les difficultés auxquelles se heurte l'analyse des offsets trouvent leur origine dans le manque de données disponibles, dans l'extrême diversité des politiques et pratiques des différents pays. Certaines confusions avec des pratiques apparentées dans le

6 Udis, Bernard [1996]. US-Swiss F-5 Transaction and the Evolution of Swiss Offset, dans Martin, Stephen (ed.) [1996], The Economics of Offsets: Defense Procurement and Countertrade, Harwood Academic Publishers, op. cit., pp. 321-335.

7 Redlich, Alon and Maison Miscavage [1996]. The Business of Offset: A Practitioner's Perspective. Case Study: Israel, dans Martin, Stephen (ed.) [1996], The Economics of Offsets: Defense Procurement and Countertrade, Harwood Academic Publishers, op. cit., pp. 381-406.

119 Economie et Institutions - n²12\&13-2008-2009 
commerce civil et la présence de forts préjugés négatifs sur ce type de politiques jouent également un rôle important.

La disponibilité de données agrégées sur les accords et les transactions d'offset est limitée à celles publiées par le Département du Commerce des Etats-Unis. Les rapports annuels publiés ne couvrent que les activités des entreprises américaines et portent sur une période qui débute en 1993. S'agissant des offsets intraeuropéens, les seules estimations existantes ont été publiées dans l'étude commandée par l'Agence Européenne de Défense. Il s'agit d'une étude ponctuelle qui ne donnera lieu à aucune mise à jour régulière.

Concernant les politiques et pratiques d'offsets, des informations concernant plus de 75 pays sont disponibles dans 1"Offset Guidelines Quarterly Bulletin" publié par Countertrade \& Offsets (CTO). Cette publication s'adresse essentiellement aux professionnels et reste relativement peu accessible et peu utilisée par les chercheurs.

Comme la compilation du CTO le montre, les politiques et pratiques d'offset des différents pays présentent une très forte diversité touchant tous les paramètres importants tel que les pourcentages d'offset requis, les secteurs visés, l'acceptabilité ou non d'offsets civils. Cette diversité a pour conséquence de réduire la clarté du débat. Elle implique aussi qu'il est illusoire de rechercher à établir une théorie unifiée des offsets : il est inapproprié de traiter de la même manière des transactions impliquant la coproduction de systèmes complexes de défense et des compensations sous forme d'achats de produits agricoles ou de services de tourisme.

Les offsets n'ayant aucun lien avec l'industrie de la défense et les secteurs reliés peuvent aussi induire une confusion entre la logique des politiques d'offset et celle de certaines pratiques de compensation civile, particulièrement populaires dans les années 1970-1980, telles que le troc et le contre-achat ${ }^{8}$. Celles-ci étaient caractéristiques du commerce Nord-Sud et Ouest-Est et avaient lieu entre des pays ayant des niveaux de développement économique et technologique différents. Leur usage possédait souvent une dimension de financement des importations par des pays possédant des systèmes stricts de contrôles des changes, de faibles réserves de devises internationales et ayant un accès limité aux marchés internationaux de capitaux. A l'opposé, les offsets liées au commerce de produits de défense ont majoritairement lieu entre des pays de

8 Le troc constitue un échange direct de biens ou service sans recours à la monnaie et fondé sur un contrat unique. Le contre-achat implique une obligation pour l'exportateur initial à acheter des biens ou des services à l'importateur initial pour une valeur déterminée en pourcentage du contrat initial et sur une durée déterminée. Il implique deux transactions séparées mais liées, avec payements monétaires.

120 Economie et Institutions - n²12\&13-2008-2009 
niveau de développement comparable et ne souffrant pas de problèmes importants en matière de financement des importations. Le Tableau 1 illustre ce constat. Il indique les 20 pays les plus importants en matière de valeur d'accords et de transactions d'offset réalisées par les entreprises de défense américaines sur la période 1993-2006.

\begin{tabular}{llll}
\hline Rang & $\begin{array}{l}\text { Top 20: Valeur des } \\
\text { Transactions } \\
\text { d'offset } \\
(1993-2006)(\mathrm{US} \\
\text { DoC [2007]) }\end{array}$ & $\begin{array}{l}\text { Top 20 : Valeur } \\
\text { des } \\
\text { Accords d'offset } \\
(1993-2006)(\mathrm{US} \\
\text { DoC [2007]) }\end{array}$ & $\begin{array}{l}\text { Index Global de } \\
\text { Compétitivité } \\
(2008-2009)(\mathrm{WEF} \\
[2008])\end{array}$ \\
\hline $\mathbf{1}$ & Royaume-Uni & Royaume-Uni & Etats-Unis ${ }^{9}$ \\
$\mathbf{2}$ & Israël & Guisse \\
$\mathbf{3}$ & Finlande & Pologne & Danemark \\
$\mathbf{4}$ & Pologne & Corée du Sud & Suède \\
$\mathbf{5}$ & Corée du Sud & Canada & Singapour \\
$\mathbf{6}$ & Italie & Pays-Bas & Finlande \\
$\mathbf{7}$ & Pays-Bas & Italie & Allemagne \\
$\mathbf{8}$ & Grèce & Taiwan & Pays-Bas \\
$\mathbf{9}$ & Canada & Israël & Japon \\
\hline $\mathbf{1 0}$ & Australie & Suisse & Canada \\
$\mathbf{1 1}$ & Suisse & Turquie & Hong Kong \\
\hline $\mathbf{1 2}$ & Espagne & Espagne & Royaume-Uni \\
$\mathbf{1 3}$ & Turquie & Australie & Corée du Sud \\
\hline $\mathbf{1 4}$ & Taiwan & Arabie Saoudite & Autriche \\
$\mathbf{1 5}$ & Norvège & Norvège \\
$\mathbf{1 6}$ & Allemagne & Danemark & France \\
\hline $\mathbf{1 7}$ & Danemark & France & Taiwan \\
$\mathbf{1 8}$ & France & EAU & Australie \\
\hline $\mathbf{1 9}$ & Belgique & Portugal & Belgique \\
$\mathbf{2 0}$ & Malaisie & Koweït & Islande \\
\hline & & & Malaisie \\
\hline
\end{tabular}

Le Tableau 1 permet aussi d'aborder, de manière critique, la présence de préjugés négatifs sur les politiques d'offset. Ceux-ci étaient présents dans l'analyse des compensations civiles et l'extension d'une telle approche aux offsets n'est pas surprenante. Les politiques d'offset sont ainsi présentées comme inefficaces, permettant de fournir des opportunités à des industries noncompétitives et, de ce fait, source de distorsions importantes dans la division internationale du travail.

9 Les Etats-Unis occupent la première place dans le classement de l'Index global de compétitivité. Cependant, étant donné que les données sur les offsets sont basées sur les contrats conclus par des entreprises américaines, les Etats-Unis ne peuvent y apparaître. Nous avons donc pris en considération les 20 pays les mieux classés après les Etats-Unis dans le Index global de compétitivité.

121 Economie et Institutions - n²12\&13-2008-2009 
Les offsets, comme sous-catégorie des compensations internationales, sont jugés contraires aux principes des accords du GATT et de l'OMC (Czinkota and Tablot [1986]), ainsi qu'aux règles du marché commun européen (FOI \& SCS [2007]). Ils sont spécifiquement prohibés par l'article XVI de l'Accord sur les marchés publics de 1'OMC. Des mesures pertinentes sont aussi présentes dans l'Accord sur les mesures concernant les investissements et liees au commerce.

Un regard rapide sur le Tableau 1 amène à un constat curieux et inattendu : la moitié des pays du Top 20 en matière de valeur des accords d'offset conclus par des entreprises américaines figure également dans le Top 20 des pays aux économies les plus compétitives sur le plan mondial du point vue de l'Index global de compétitivité établi par le World Economic Forum ${ }^{10}$. Si l'on se base sur le classement en termes de valeur de transactions d'offset réalisées, la proportion passe à 14 pays sur 20 .

Nous nous trouvons donc confrontés à une contradiction importante : les pays les plus compétitifs au monde recourent à des politiques d'offset pour fournir des opportunités industrielles à leurs entreprises nationales. Deux questions importantes se posent alors. En premier, pourquoi les relations dont ces politiques visent à favoriser l'émergence ne s'établissent pas spontanément? Plus particulièrement, il est nécessaire de s'interroger sur les barrières qui empêchent les entreprises efficaces et fiables des industries de défense des pays importateurs de devenir fournisseurs des grands intégrateurs de systèmes mondiaux. Ensuite, est-il acceptable d'affirmer que les politiques d'offset puissent connaître un tel succès sur le long terme si elles étaient complètement inefficaces, ou efficaces uniquement sur le court terme au prix de fortes distorsions dans la structure du commerce international ? Ceci est d'autant plus important qu'il est ici question de politiques pratiquées par des pays réputés comme ayant aussi des structures de gouvernance comptant parmi les plus efficaces au monde.

A la première de ces questions, la théorie traditionnelle du commerce international, en grande partie responsable de la vision négative dominante sur les offsets, ne peut apporter qu'une réponse partielle. Le facteur pouvant empêcher l'établissement de partenariats industriels efficaces est le protectionnisme. Les offsets peuvent ainsi être considérés comme une politique de rétorsion commerciale permettant de forcer les pays dans lesquels les grands intégrateurs de systèmes de défense sont localisés à temporairement abaisser leur protection nationale dans un cadre bilatéral. Cette explication capture une partie de la réalité comme le montre

10 World Economic Forum [2008], The Global Competitiveness Report 20082009.

122 Economie et Institutions - n²12\&13-2008-2009 
l'exemple du programme d'offsets rattaché à la vente des F-5 à la Suisse. Cependant, cela ne peut rendre compte du maintien des relations ainsi établies au-delà de la durée du programme d'offset. A la fin de celui-ci les mesures protectionnistes devraient redevenir opérationnelles et rompre les relations établies. Or, comme nous l'avons souligné précédemment, les offsets peuvent aboutir à l'établissement de relations durables capables de survivre à la fin du programme d'offset. Dans certains cas des relations peuvent même s'établir indépendamment de l'obtention du marché public auquel les obligations d'offset sont rattachées. Il est évident que la théorie traditionnelle du commerce internationale ne permet pas d'apporter une explication satisfaisante à ces phénomènes. Une approche plus fine, prenant en compte les facteurs institutionnels et s'inscrivant dans une optique de politique industrielle est donc nécessaire.

\section{Section 2: Une approche en termes d'économie industrielle : le rôle des coûts de changements et des externalités de R\&D}

Comprendre les mécanismes d'offset nécessite de se placer dans une grille de lecture en termes d'économie industrielle. En effet, si les offsets touchent des transactions commerciales au niveau international, il n'en demeure pas moins qu'une lecture en termes de théorie classique du commerce international est insuffisante. D'une part, la théorie classique du commerce international couvre faiblement les biens complexes et les biens systèmes comme ceux de l'armement que nous cherchons à décrire. D'autre part, les motivations en termes de structuration de l'industrie et des retombées technologique des offsets n'est pas mentionnée alors même qu'elle pourrait être la source même de ces opérations. Les avantages comparatifs et compétitifs ne sont pas suffisants à expliquer la configuration de la division internationale du travail. Nous proposons dans cette section d'expliquer le rôle des coûts de changement et des externalités de R\&D dans la mise en place des offsets au niveau international.

\section{a) Les coûts de recherche et de changements de fournisseurs comme facteur explicatif des offsets}

La production de biens d'armement pourrait être considéré comme la production d'un bien modulaire (Steinmueller [2005]). Cette logique modulaire a démontré sa supériorité industrielle et financière ses dernières années sur d'autres formes d'organisation de la production dans de nombreux secteurs industriels comme l'Automobile, l'Aérospatiale, l'Informatique et le grand électronique. Ainsi, une firme (pays) contrôlerait l'intégration de nombreux

123 Economie et Institutions - n $12 \& 13-2008-2009$ 
composants dans la production du bien système. La viabilité du système et sa compétitivité dépendent en grande partie de la capacité du coordonateur à maîtriser les coûts de production (compétitivité prix) et à utiliser des technologies avancées (compétitivité technologique). Les deux logiques combinées débouchent sur la recherche des meilleures entreprises permettant d'apporter à la fois en termes de compétitivité prix et hors prix.

Dans le secteur de la défense les partenariats industriels se sont traditionnellement construits dans le cadre national. Une fois que ces relations sont établies, les partenaires en place jouissent d'une position privilégiée. Ils sont protégés par l'existence d'un différentiel de coûts de transaction qu'il est possible d'analyser à travers le concept de coûts de changement de fournisseurs. Les coûts de changement peuvent être définis comme "les coûts réels ou perçus supportés en cas de changement de fournisseur et qui ne sont pas supportés en continuant les relations avec le fournisseur en place " (OFT [2003]). Ils sont le résultat d'investissements spécifiques au fournisseur présent et qui doivent être dupliqués pour un nouveau fournisseur (Farrell \& Klemperer [2004])

L'analyse des effets des coûts de changement a été développée dans le cadre de l'étude du comportement de consommation. Les concepts développés peuvent cependant être facilement transposés à l'analyse des relations industrielles. Une typologie des coûts de changement adaptée au sujet qui nous intéresse peut être présentée. Celle-ci couvre les coûts liés aux imperfections informationnelles, traditionnellement appelés coûts de recherche, et les différentes formes de coûts de changement, au sens restreint du terme, inspirés de la liste établie par Klemperer [1995].

Les coûts résultants des imperfections informationnelles: "awareness" et coûts de recherche

Le choix concernant le changement de fournisseurs est hautement influencé par la présence d'imperfections informationnelles. D'après Giulietti, Waddams Price et Waterson [2005], une première contrainte fondamentale vient la conscience même de la possibilité de changement de fournisseur, appelée "awareness". Si cette dernière n'est pas présente, aucune recherche de nouveaux fournisseurs n'aura lieu. Giulietti, Waddams Price et Waterson [2005] s'intéressent au comportement des consommateurs individuels d'énergie et le concept n'est donc pas directement transposable. Cependant, l'idée demeure raisonnablement pertinente dans le cadre de l'industrie de la défense, où il est plutôt question de la conscience de l'existence de fournisseurs plus efficaces que ceux déjà en place. Ce problème peut être fortement exacerbé lorsque l'on considère la possibilité de recourir à des entreprises étrangères : une certaine tendance à croire en l'excellence de l'industrie nationale a

124 Economie et Institutions - n $12 \& 13-2008-2009$ 
toujours été présente dans le secteur de la défense. De plus, la présence de marchés nationaux captifs réduit la pression concurrentielle et l'acuité de la nécessité d'accroître l'efficacité économique de la production.

Si la contrainte liée à l'uawareness" est remplie, des coûts de recherche doivent néanmoins être supportés afin d'obtenir des informations sur des fournisseurs potentiels et leurs caractéristiques telles que les prix, la qualité, la localisation, les capacités... Ces coûts couvrent les coûts de la collecte d'information, mais aussi des coûts implicites comme la valeur du temps ou les coûts d'opportunité. Un agent rationnel faisant face à des coûts de recherche ne cherchera pas à obtenir toutes les informations possibles sur tous les fournisseurs potentiels. Si les coûts de recherche sont suffisamment larges relativement aux gains espérés de la recherche les relations avec les fournisseurs en place seront automatiquement reconduites. Dans le cadre des relations industrielles les coûts de recherche peuvent jouer un rôle particulièrement important en ce qui concerne les composants de technologie moyenne ou modérément forte. Pour les produits de base et les matières premières des marchés internationaux intégrés existent souvent renduisant au minimum la nécessité de mener une recherche. Pour les produits de très haute technologie la situation peut-être comparable en raison du très faible nombre de fournisseurs potentiels disponibles au niveau mondial. Entre ces deux extrêmes cependant, des nombreux fournisseurs potentiels existent qui peuvent être mal connus, voir même complètement inconnus, par les intégrateurs de système. Les activités de recherche acquièrent alors une forte importance. Naturellement, il en va de même pour les coûts qui les accompagnent.

\section{Coûts de compatibilité}

La complexité croissante des produits de défense, leur caractère systémique et le rôle accru que joue l'électronique sont de nature à exacerber les coûts nécessaires pour garantir la compatibilité. L'introduction de la conception assistée par ordinateur peut en limiter partiellement les effets, mais en contrepartie il existe également des risques importants liés aux difficultés concernant l'établissement des responsabilités des différents partenaires en cas de mauvais fonctionnement d'un bien complexe (Steinmueller [2005]). Les procédures spécifiques entre partenaires visant à garantir la compatibilité et à régler les difficultés pouvant apparaître en cas d'échec sont autant de sources de coûts, implicites ou explicites, au changement de fournisseurs

Coûts de transaction liés au changement de fournisseurs. 
Etablir des relations avec de nouveaux partenaires réclame que des négociations soient menées et de nouveaux contrats écrits. La mise en place de nouveaux circuits financiers et de structures de coordination sera souvent nécessaire. Des coûts de transaction en résultent, qui sont d'autant plus forts que les produits ou composants concernés sont complexes et que les relations qui s'établissent inscrites dans le long terme.

\section{Coûts d'apprentissage}

Les relations avec de nouveaux fournisseurs impliquent un processus d'apprentissage aussi bien organisationnel que technologique. La construction d'une interface efficace entre partenaires implique non-seulement des coûts explicites, mais aussi des coûts implicites liés aux délais induits, à des échecs pouvant potentiellement survenir lors des premières phases de la collaboration. Lorsqu'il s'agit de relations transfrontalières les difficultés peuvent être exacerbées par des différences linguistiques, culturelles ou relatives aux pratiques d'organisation industrielle.

\section{Coûts liés à l'incertitude (sur la qualité)}

Le processus d'intégration de composants complexes implique des risques technologiques importants: la défaillance d'un seul composant peut mettre en cause le fonctionnement du système final entier. L'incertitude sur la qualité et la compatibilité des produits de nouveaux fournisseurs peut constituer un frein important au changement de partenaires. Qui plus est, la complexité technologique et les difficultés rencontrées pour établir les responsabilités en cas de mauvais fonctionnement impliquent aussi des risques accrus liés aux comportements opportunistes. La confiance générée grâce à des relations durables et suivies peut alors empêcher l'entrée de nouveaux fournisseurs dans la chaîne d'approvisionnement des intégrateurs de systèmes.

\section{Coûts de changement contractuels}

Les coûts de changement contractuels sont des coûts de changement artificiels induits en particulier par la présence de relations contractuelles entre partenaires. La présence de contrats de long terme en constitue un exemple. Ce type de coûts peut naître en dehors d'une quelconque intention délibérée. Ils peuvent aussi être le résultat d'une action volontaire visant, dans le cadre de la mise en place des relations contractuelles, à se construire une protection fondée sur les coûts de changement.

La présence de ce type de coûts peut avoir pour effet de limiter la recherche de nouveaux fournisseurs. De plus, les coûts de recherche et de changement de fournisseurs peuvent aussi être à l'origine d'un effet d'hystérèse des politiques protectionnistes

126 Economie et Institutions - n $12 \& 13-2008-2009$ 
(Hartigan [1996], Greaney [2000]), les effets de celles-ci continuant à s'exercer après la suppression des réglementations restrictives.

Les offsets peuvent alors intervenir, en tant que système combinant une incitation à rechercher des partenaires dans les pays importateurs à cause du conditionnement du contrat de base, et des profits qui en sont tirés, et d'une subvention implicite payée par le gouvernement importateur (Ianakiev [2005]). Cette subvention résulte du fait que les surcoûts induits par les offsets sont généralement intégrés au prix du contrat initial et supportés par le gouvernement importateur (Hartley [1995a]). Ceci représente une subvention des coûts de recherche et changement dans les cas où de nouveaux fournisseurs efficaces sont découverts.

En effet, d'un strict point de vue industriel, les entreprises d'armement sont obligées de rechercher des entreprises efficaces afin de les intégrer dans la production du bien complexe.

Cependant, l'intégration de nouvelles firmes dans le système de production nécessite également de considérer le volet technologique. La R\&D militaire occupe une place centrale dans les dépenses de R\&D d'une nation et la compétitivité technologique est fondamentale dans un contexte de forte concurrence. Une lecture des offsets selon cette logique s'avère assez instructive.

\section{b) Externalités de R\&D et opérations d'offsets}

La seconde caractéristique de la logique industrielle des offsets concerne les externalités de R\&D qu'elles génèrent. En effet, les pays importateurs sont fortement intéressés par les retombées technologiques et par les possibilités de rattrapage technologique. Le pays producteur (dans lequel l'intégrateur du bien militaire est basé) est intéressé quant à lui par de nouvelles possibilités industrielles et technologiques que peuvent lui apporter les nouveaux pays importateurs. L'examen attentif des opérations d'offsets montre que les accords des firmes dépassent la période négociée initialement. Ceci tend à démontrer que le pays exportateur a pu soit mettre à niveau les entreprises partenaires, soit bénéficier d'un apport technologique inattendu.

La montée en puissance des offsets dans les opérations de commerce d'armement démontre globalement un changement dans la logique d'innovation dans le secteur militaire. Pour des auteurs comme Versailles et Mérindol [2006], les opérations d'offsets pourraient s'interpréter comme un changement d'approche dans les programmes de R\&D militaires d'une logique concurrentielle à une logique coopérative. Alors que la recherche et l'innovation étaient tournées vers un système fermé on constate que cette logique a atteint ses limites et le secteur d'armement se tourne de nos jours

127 Economie et Institutions - nº $12 \& 13-2008-2009$ 
davantage vers une logique d'innovation ouverte (Le Texier, 2008)11. L'établissement des offsets permet alors aux pays participants d'adhérer à des "systèmes internationaux technologiques " dépassant leur cadre national.

Les opérations d'offsets permettraient ainsi aux pays de maintenir leur système d'innovation ouvert et de capter une partie des retombées technologiques internationales. De manière générale, on constate l'émergence d'un nouveau paradigme militaire ${ }^{12}$ centré sur les technologies de la connaissance. L'impact des technologies militaires dépasse ainsi le cadre standard des armes pour couvrir des domaines économiques plus vastes (Technologies de l'Information, Intelligence Economique...). Ceci assurerait une meilleure compétitivité13.

Cette stratégie a été poursuivie par de petits pays comme Israël, la Suède ou encore 1'Australie. Qui ont pu développer des armes sophistiquées dans certaines niches en s'impliquant dans des programmes d'offsets au niveau international avec les Etats-Unis ou l'Angleterre. De nombreux pays aspirent à imiter cette stratégie et les offsets permettent ainsi de s'impliquer dans un système technologique compétitif et de disposer de savoirs faire importants.

"Increasingly, small countries are net direct and indirect importers of military technology as buyers of complete weapons systems or their components, or importers of designs and disembodied technological know-how. But the trade in military know-how is rather complicated in part because not all new knowledge developed by say, the USA, is available even to its closest allies and, in part, because what is available can often only be accessed using complex mechanisms involving international (R\&D) collaborative agreements, barter trade and/or technological offsets (Brauer and Dunne, [2004])."

11 Le Texier [2008] démontre cette assertion dans le cadre de l'innovation relative au secteur du logiciel.

12 In the last decade, however, many countries, large and small, have been attracted to the apparent benefit to be gained by exploiting the range of knowledge-intensive military technologies that have become known as the Revolution in Military Affairs (RMA). Adopting the RMA war-fighting paradigm involves a shift away from relatively self-contained 'weaponcentric' technologies to knowledge-intensive, digital and network-enabled weapons systems. These require massive complementary investments in intelligence, communications, command and control systems to enable component weapons systems to work synergistically as an integrated array of interoperative and interdependent military assets. [Whyle et al. 2006].

13 L'impact de la R\&D militaire sur le secteur civil demeure un sujet hautement controversé. Pour de nombreux auteurs, ces retombées sont limitées.

128 Economie et Institutions - n²12\&13-2008-2009 
De manière globale, la problématique des offsets se situe dans les prolongements sur les débats portant sur les externalités technologiques du secteur de la défense et la manière de les transposer dans le secteur civil. Les effets de "spins-offs" sont recherchés par les pays importateurs en essayant de stimuler simultanément le secteur militaire et surtout civil. Ainsi, les orientations des opérations d'offsets peuvent être d'emblée tournées vers la recherche d'externalités de recherche et développement. Mais les problèmes relatifs aux droits de propriété intellectuels limitent fortement cette approche (Bellais \& Guichard [2006], Le Texier [2008]).

Ainsi, cette section nous a permis de souligner la logique industrielle des opérations d'offsets. Elles sont ainsi un instrument à part entière des politiques industrielles poursuivies par certaines nations en quête de rattrapage technologique et d'insertion dans des systèmes de production plus compétitifs. Alors que les pays exportateurs établissent des partenariats technologiques sous des contraintes de coûts de recherche élevés, les pays importateurs cherchent à optimiser les externalités de R\&D et l'insertion dans des système international d'innovation. Les contrats d'offsets peuvent aboutir à une situation de donnant-donnant voire même de gagnantgagnant. Dans ce contexte, comment peut-on prendre en compte leur rôle industriel structurant dans l'espace économique européen ?

\section{Section 3: Quelles implications pour l'Europe de la défense?}

Les efforts visant à créer un marché intégré des équipements de défense dans l'Union Européenne vont se heurter aux effets combinés d'un protectionnisme toujours important et de barrières naturelles à l'établissement de partenariats industriels transfrontaliers. La contribution des offsets n'est alors pas univoque : ils peuvent être à l'origine de distorsions importantes de la division internationale du travail, mais ils peuvent aussi agir en tant que facteur d'intégration.

Le risque de détournement de trafic inhérent à l'usage des politiques d'offset a été largement discuté dans la littérature économique. On peut aisément montrer que la conditionnalité du contrat de base, et des profits qui en résultent, par rapport à l'établissement de relations avec des entreprises locales induit des incitations suffisamment puissantes, pour que des partenaires inefficaces puissent être choisis (Ianakiev [2005]). Ceci est d'autant plus probable que les surcoûts induits sont facturés à l'importateur. 
Néanmoins, les incitations résultant du lien établi entre le contrat de base et les offsets peuvent aussi pousser à la recherche et à la découverte de partenaires efficaces et mener à l'établissement de relations durables. L'effet est aussi soutenu par la subvention implicite des coûts de recherche et de changement qui est opérée par le gouvernement acheteur.

La découverte de fournisseurs étrangers performants suite aux accords d'offset joue aussi un rôle important en matière de prise de conscience, d' "awareness". Une fois que les entreprises situées dans les grands pays producteurs de systèmes d'armement ont clairement constaté la présence de partenaires potentiels efficaces en dehors de leur cadre national, ou dans des pays dans lesquels ils n'étaient pas habitués à travailler, ce type particulier de barrière de type informationnel est fortement réduit. L'effet sera renforcé si des expériences positives sont accumulées dans le cadre de multiples transactions concernant des pays différents.

Les politiques d'offset peuvent donc être un facteur favorisant l'intégration du marché européen. Cette possibilité est reconnue par l'étude sur les offsets commandée par l'AED. Il est alors approprié de considérer les offsets comme un symptôme des déficiences du marché européen des équipements de défense, en particulier des barrières à l'accès aux chaînes d'approvisionnement des grands intégrateurs de systèmes, plutôt que comme un problème indépendant (Ianakiev et Mladenov [2008]). Les offsets sont une conséquence de l'inexistence d'un marché intégré, et c'est l'établissement de ce-dernier qui devrait permettre d'en limiter l'usage.

Cependant, comme les offsets peuvent aussi exercer des effets négatifs, il est utile de prendre des mesures permettant d'en limiter l'usage excessif et les distorsions pouvant en résulter. Une telle mesure est le Code de Conduite en matière d'offsets élaboré par $1^{\prime} A E D^{14}$. Il est également important de mettre en évidence d'autres mesures, alternatives ou complémentaires, qui peuvent permettre de favorise l'émergence d'un véritable marché européen des produits de défense.

\section{Préférences nationales et marché européen de la défense}

Limiter au maximum le protectionnisme national constitue un préalable important. La fragmentation du marché des produits de défense en Europe est largement due au maintien de préférences nationales basées sur un usage extensif, voir même abusif, de l'article 296 du Traité CE. Celui-ci introduit une dérogation au

14 http:/ / www.eda.europa.eu/genericitem.aspx?area $=28 \mathrm{id}=420$

130 Economie et Institutions - n²12\&13-2008-2009 
respect des règles du marché intérieur en ce qui concerne la production et le commerce d'armements, dérogation fondée sur la protection des "intérêts essentiels de sécurité" des Etats membres ${ }^{15}$. Pendant des décennies les dispositions de l'article 296 ont été utilisées en tant qu'exception générale et automatique permettant de mettre à l'abri des règles sur les achats publics la quasi-totalité des commandes effectuées par les ministères de la défense ou les agences d'armement. Il a fallu attendre les années 1990 pour qu'une jurisprudence de la Cour de Justice des Communautés Européennes apparaisse, affirmant qu'un tel usage de l'article 296 était contraire à l'esprit du Traité CE. D'après la cour l'article doit être interprété de manière restrictive, son usage devant être justifié au cas par cas et limité aux situations mettant effectivement en jeu les "intérêts essentiels de sécurité ". Dans une Communication interprétative publiée en 200616, la Commission européenne a résumé la jurisprudence sur l'application de l'article 296, affirmant ainsi son intention de défendre les principes définis par la Cour.

Un obstacle majeur demeure cependant quant à la prise d'actions fermes dans ce sens : les règles relatives aux achats publics en vigueur dans l'UE sont reconnues, y compris par la Commission, comme peu adaptées au domaine de l'armement. C'est pour palier à ce problème que la Commission a proposé en 2007 une Directive spécifique régulant les achats publics dans le domaine de la défense et de la sécurité17. Le texte proposé doit permettre de combiner l'introduction d'un haut degré de transparence et de compétition

15 Article 296 du Traité CE :

"(1)Les dispositions du présent traité ne font pas obstacle aux règles ci-après:

a) aucun État membre n'est tenu de fournir des renseignements dont il estimerait la divulgation contraire aux intérêts essentiels de sa sécurité;

b) tout État membre peut prendre les mesures qu'il estime nécessaires à la protection des intérêts essentiels de sa sécurité et qui se rapportent à la production ou au commerce d'armes, de munitions et de matériel de guerre; ces mesures ne doivent pas altérer les conditions de la concurrence dans le marché commun en ce qui concerne les produits non destinés à des fins spécifiquement militaires."

16 Commission Européenne [2006], Communication Interprétative sur l'application de l'article 296 du traité dans le domaine des marché publics de la défense, COM (2006) 779.

17 Commission Européenne[2007], Proposition de Directive relative à la coordination des procédures de passation de certains marchés publics de travaux, de fournitures et de services dans les domaines de la défense et de la sécurité, $\operatorname{COM}(2007) 766$.

131 Economie et Institutions - n²12\&13-2008-2009 
dans les procédures d'achats de produits de défense tout en garantissant la flexibilité nécessaire au secteur. Des facteurs importants tels que la sécurité de l'information et de l'approvisionnement sont également pris en compte.

L'adoption du texte va constituer un pas important dans la construction d'un marché européen des équipements de défense. La présence de la nouvelle directive devrait avoir pour effet de restreindre le recours à l'article 296, l'absence de régulation adaptée aux achats publics d'armement ne pouvant plus être invoquée.

Aux actions prises par les institutions communautaires viennent également s'ajouter les initiatives prises au sein de l'Agence Européenne de Défense, en particulier le Code de conduite en matière d'achats publics de défense adopté en novembre $2005^{18}$. Bien que non-contraignant sur le plan légal, celui-ci constitue un facteur supplémentaire dans la réduction des barrières protectionnistes en vigueur.

Cependant, comme cela a été avancé dans les développements précédents, la simple suppression des mesures protectionnistes n'est pas suffisante dans une situation ou des coûts de transaction importants sont présents et où, en conséquence, les événements historiques marquent la structure industrielle de manière durable.

\section{Rôle des pratiques des acteurs privés}

Dans un tel cadre la construction d'un marché intégré des équipements de défense va nécessiter la mise en place de mesures permettant de limiter l'influence des barrières naturelles à l'établissement de partenariats industriels transfrontaliers, et en particulier celle des imperfections informationnelles et des coûts relatifs au changement de fournisseurs.

Un premier enjeu concerne la gestion des chaînes d'approvisionnement par les entreprises de défense. La structure industrielle du secteur a été forgée essentiellement dans un cadre national. Pour qu'un mouvement d'internationalisation et d'intégration européenne puisse avoir lieu il est nécessaire que les entreprises du secteur adoptent une approche de recherche active de fournisseurs hors de leurs économies nationales. L'étude commandée par l'AED met spécifiquement l'accent sur le rôle que peut jouer l'introduction de pratiques modernes de gestion des

18http://www.eda.europa.eu/genericitem.aspx?area=Organisation\&id=154

132 Economie et Institutions - n²12\&13-2008-2009 
chaînes d'approvisionnement afin de réduire la nécessité de recourir aux offsets en tant qu'instrument d'accès au marché. On ne peut contester que l'industrie de la défense n'est pas toujours réputée pour ses techniques de gestion performantes et qu'une certaine résistance à l'ajustement y est présente (Braddon \& Dowdall [1996], Dowdall [2004]). Il faut noter que le secteur connait une internationalisation croissante et qu'une part importante des grands intégrateurs de systèmes est constituée de firmes, telles que Boeing, ayant des activités civiles très importantes. Etant donné la pression concurrentielle qui s'exerce sur les activités duales, et la pression sur les coûts associée aux budgets de Défense, la gestion des programmes d'armement a été rendue plus performante avec la mise en place de pratiques de gestion sophistiquées. Quand le recul sur ces sujets le permettra, il sera pertinent de mesurer comment les divisions militaires en auront bénéficié.

Par ailleurs, une recherche limitée de partenaires étrangers n'est pas obligatoirement synonyme de mauvaise gestion des chaînes d'approvisionnement : en présence d'importants coûts de recherche et de changement il peut s'agir d'une réponse parfaitement rationnelle et cohérente avec les prévisions de la théorie économique. Si tel est le cas, l'introduction de pratiques modernes de gestion de chaînes d'approvisionnement pourrait être insuffisante pour faciliter l'établissement de partenariats industriels transfrontaliers.

Il est donc nécessaire de s'attaquer directement au problème de fond: la présence de coûts de transaction, plus particulièrement de coûts de recherche et de changement de fournisseurs. Des mesures peuvent être prises pour réduire les coûts informationnels.

Une approche traditionnelle dans ce domaine consiste à organiser des expositions et des séminaires d'industrie et de technologie destinés à assurer la promotion de l'industrie de défense d'un pays ou d'une région. Ces séminaires souffrent cependant du fait qu'ils sont articulés autour de "ce que les compagnies locales désirent vendre "19. Or, d'après Redlich $\&$ Miscavage [1996], le succès des initiatives comparables ayant lieu dans le cadre de la préparation de certains programmes d'offset réside dans le fait qu'ils sont structurés autour des grands intégrateurs de système, de ce qu'ils désirent acheter et des besoins et enjeux stratégiques auxquels ils font face. Dans ce domaine, un rôle constructif peut être joué par des associations internationales d'industrie telles que l'European Aerospace and Defence Industry Association (ASD). Elles se présentent comme l'interface naturelle permettant de mettre des entreprises de pays et de tailles différentes en relation. Elles doivent

19 Redlich, Alon and Maison Miscavage [1996]. op. cit., p. 403.

133 Economie et Institutions - n²12\&13-2008-2009 
également être en mesure de personnaliser les activités organisées afin qu'elles correspondent suffisamment aux exigences des différentes parties concernées.

\section{Coûts d'information et de changement et intégration du marché européen de la défense}

Une autre manière de réduire les coûts d'information consiste à mettre en place des systèmes de centralisation des informations sur les opportunités de sous-traitance. Un tel système est actuellement opérationnel au sein de l'AED. Depuis le mois de mars 2007 une partie de l'Electronic Bulletin Board de l'agence est dédié à la publication d'avis d'opportunités d'achats par les entreprises de défense dans le cadre de l'application du code de meilleures pratiques dans les chaînes d'approvisionnement. La plateforme jouit du soutien de l'ASD et, bien que jusqu'à présent elle n'ait connu qu'un succès limité, elle a le potentiel d'être un facteur d'intégration non-négligeable.

Cette dynamique devrait être considérablement renforcée par la nouvelle Directive européenne sur les achats de produits de défense qui prévoit la possibilité, pour l'Etat acheteur, d'exiger qu'une part du contrat, n'excédant pas $30 \%$, soit sous-traitée. Le choix des sous-traitants devra être le résultat d'une procédure compétitive et ouverte à toute entreprise européenne. Les opportunités résultant de cette procédure devraient permettre une ouverture croissante des chaînes d'approvisionnement des grands groupes de défense et les avis publiés en conséquence pourraient à terme venir nourrir l'Electronic Bulletin Board de l'AED. De plus, comme dans le cadre des offsets, une subvention implicite des coûts de transaction liés à l'obligation de sous-traitance sera probablement effectuée.

Enfin, la remise à l'ordre du jour des programmes de coopération au sein de l'UE, constitue l'alternative la plus crédible aux offsets. Ils permettent de prévoir la participation industrielle et technologique des différents pays membres dès l'origine du programme. L'instabilité inhérente résultant des offsets, qui sont par nature liés à des contrats d'achat spécifiques, est ainsi évitée. De plus, les coûts du programme peuvent être réduits à condition que le principe traditionnel du "juste retour ", établissant un lien direct entre contribution financière au programme et participation industrielle, soit abandonné. Dans la perspective d'un marché intégré des équipements de défense en Europe, une logique économique fondée sur les performances des fournisseurs doit prévaloir. 
Des institutions spécialisées dans la gestion de programmes de coopération dans le domaine de l'armement ont déjà élaboré des systèmes plus flexibles que celui du juste retour traditionnel. L'OCCAR a par exemple développé un principe de juste retour global fondé sur un équilibre pluriannuel et multiprogramme, permettant une flexibilité accrue et une meilleure prise en compte des performances économiques au sein de chaque programme.

Les organismes responsables de la gestion des programmes de coopération doivent accorder une importance particulière aux barrières résultant des coûts de transaction relatifs à l'établissement de partenariats industriels tels que les coûts de recherche et de changement de fournisseurs. Dans le cadre des programmes coopératifs les poids financier de ces coûts peut être mutualisé.

\section{Conclusion}

Dans cet article nous avons cherché à caractériser le phénomène des offsets et à comprendre leur logique industrielle et leur rôle dans le processus d'intégration du marché européen des équipements de défense. Principalement, les opérations d'offsets ont été considérés ici sous l'angle de la minimisation des coûts et la cohérence technologique. De nombreux coûts sont associés à la production des biens systèmes (biens modulaires) comme le sont en général les biens d'armement. Les opérations d'offsets pourraient contribuer à la minimisation de ces coûts. Ceci permettrait d'expliquer la persistance et l'augmentation de ce phénomène durant les deux dernières décennies. La construction européenne d'un marché consolidé des produits d'armement nécessite de prendre en compte cette caractéristique afin d'accroître l'efficacité et l'efficience européenne. Mais le protectionnisme actuel et la forte implication de nombreux pays européens dans des logiques d'offsets extracommunautaires depuis le milieu des années 1970 conduit à se poser la question de l'intégration d'un tel marché.

Par ailleurs, les opérations d'offsets s'accompagnent généralement de transferts technologiques importants. Elles sont au centre des incitations des pays importateurs. L'intégration du marché européen de la défense pourrait conduire à accélérer l'Europe technologique et les synergies européennes. Ce motif est suffisant pour généraliser la pratique des offsets au sein des pays européens et les favoriser. La dynamique de recherche de partenaires transfrontaliers pourrait accroître la productivité des industries d'armement européen. Ceci pourrait passer par la mise en place d'un système de préférence communautaire. Mais ceci aura pour conséquence d'accroître l'identité européenne en matière de défense et pourrait avoir comme implication également le passage à un système d’innovation fermée (tournée vers les pays européens).

135 Economie et Institutions - $\mathrm{n}^{\circ} 12 \& 13-2008-2009$ 


\section{Bibliographie}

Braddon, D. et Dowdall, P. 1996. Flexible Networks and the Restructuring of the Regional Defence Industrial Base: the case of South West England. Defence and Peace Economics, 7, pp. 47-59.

Brauer, Jurgen and J. Paul Dunne eds. 2004. Arms Trade and Economic Development. London and New York: Routledge.

Commission Européenne. 2007a. Proposition de Directive relative à la coordination des procédures de passation de certains marchés publics de travaux, de fournitures et de services dans les domaines de la défense et de la sécurité. $\operatorname{COM(2007)~} 766$.

Commission Européenne. 2007b. Commission staff working document Accompanying document to the Proposal for a Directive on the coordination of procedures for the award of certain public works contracts, public supply contracts and public service contracts in the fields of defence and security. SEC(2007) 1598.

Commission Européenne. 2006. Communication Interprétative sur l'application de l'article 296 du traité dans le domaine des marché publics de la défense. COM (2006) 779.

Czinkota, Michael and Anne Talbot .1986. GATT Regulation of Countertrade: Issues and Prospect. The International Trade Journal. 1:2, pp. 155-172.

Dowdall, Paul. 2004. "Chains, Networks, and Shifting Paradigms: The UK Defence Industry Supply System." Defence and Peace Economics, 15:6, pp. 535-50.

Farrell, Joseh and Paul Klemperer. 2004. "Coordination and Lock-In: Competition with Switching Costs and Network Effects." preliminary draft for Handbook of Industrial Organization vol. 3.

FOI et SCS. 2007. Study on the Effects of Offsets on the Development of a European Defence Industry and Market.

Gates, Eric. 2004. "The Defence Firm of the Future." Defence and Peace Economics, 15:6, pp. 509-17.

Giulietti, Monica, Catherine Waddams Price and Michael Waterson. 2005. "Consumer Choice and Competition Policy: A Study of UK Energy Markets". The Economic Journal, 115, pp. 949-968.

Greaney, Theresa. 2000. Righting past wrongs: can import promotion policies counter hysteresis from past trade protection in the presence of switching costs? Japan and the World Economy, 12, pp. 211-227.

Hall, Peter and Stefan Markowski. 1994. "On the Normality and Abnormality of Offsets Obligations." Defence and Peace Economics, 5:3, pp. 173-88.

Hall, Peter and Stefan Markowski. 1996. "Some Lessons from the Australian Defense Offsets Experience." Defence Analysis, 12:3, pp. 289-314.

136 Economie et Institutions - nº $12 \& 13-2008-2009$ 
Hartigan, James. 1996. Perverse Consequences of the GATT Export Subsidies and Switching Costs. Economica, 63-249, pp. 153161.

Hartley, Keith. 1998. "Defence Procurement in the UK." Defence and Peace Economics, 9, pp. 39-62.

Hébert, Jean -Paul. 1998. "Les exportations d'armement. A quel Prix?" Notes et Etudes Documentaires: 5080.

Ianakiev, Gueorgui et Nickolay Mladenov. 2008. " Offset Policies in Defence Procurement: Lessons for the European Defence Equipment Market". Défense nationale et sécurité collective, Hors Série 2008.

Ianakiev, Gueorgui. 1995. How Do Offset Policies Affect the International Division of Labour? The Case of the Defence Industry. Ninth Annual International Conference on Economics and Security. Bristol.

Kirkpatrick, David. 1995. "The Rising Cost of Defence Equipment - The Reasons and the Results." Defence and Peace Economics, 6, pp. 263-88.

Kirkpatrick, David. 2004. "Trends in the Costs of Weapon Systems and the Consequences." Defence and Peace Economics, 15:3, pp. 259-73.

Klemperer, Paul. 1987. "Markets with Consumer Switching Costs." Quarterly Journal of Economics, 102:2, pp. 375-94.

Klemperer, Paul. 1987. "The Competitiveness of Markets with Switching Costs." RAND Journal of Economics, 18:1, pp. 137-50.

Klemperer, Paul. 1995. "Competition when Consumers have Switching Costs." Review of Economic Studies, 62:4, pp. 515-39.

Le Texier .2008. "Le rôle des innovations ouvertes dans la reconstruction des activités industrielles", Thèse de doctorat ès Sciences Economiques, Université de Nice Sophia-Antipolis.

Markowski, Stefan. 1998. "Switzerland - The Pragmatic Approach to Defence Procurement." Defence and Peace Economics, 9, pp. 99-118.

Martin, Stephen. 1996. The Economics of Offsets: Defense Procurement and Countertrade: Harwood Academic Publishers.

Martin, Stephen and Keith Hartley. 1995a. "Defense Equipment, Exports and Offsets: The UK Experience." Defence Analysis, 11:1, pp. 21-30.

Martin, Stephen and Keith Hartley. 1995b. "UK Firms' Experience and Perceptions of Defence Offsets: Survey Results." Defence and Peace Economics, 6, pp. 123-39.

Matthews, Ron. 1991. "Offsets to Decline for Britain's Defence-Industrial Base ?" The RUSI Journal, 136:4, pp. 58-64.

Neuman, Stephanie. 1985. "Coproduction, Barter, and Countertrade: Offsets in the International Arms Market." Orbis, 29, pp. 183-213.

137 Economie et Institutions - n²12\&13-2008-2009 
Office of Fair Trading. 2003. "Switching Costs." Economic Discussion Paper.

Pugh, Philip. 1986. The Cost of Seapower. London: Conway Maritime Press.

Pugh, Philip. 1993. "The Procurement Nexus." Defence Economics, 4, pp. 179-94.

Sauvin, Thierry. 1991. "La compensation internationale: du troc à la coopération internationale." Université Paris X.

Sauvin, Thierry. 1995. "Modalités et fonctions des accords de compensation internationaux : le cas des relations Nord-Sud." Tiers Monde,octobre - décembre, pp. 897-912.

Sauvin, Thierry. 1992. "La compensation, un nouveau moyen d'intervention de l'Etat dans les pays industrialisés." Cahiers d'économie mondiale, janvier-juin, pp. 37-48.

Sauvin, Thierry. 1993. "La compensation internationale : un nouveau mode de coordination des économies au sein de l'économie mondiale." Economies et sociétés: Juin, pp. 57-74.

Stiglitz, Joseph E. 1989. "Imperfect Information in the Product Market." in Handbook of Industrial Organization. Richard Schmalensee and Robert Willing eds, pp. 769-847.

Struys, Wally. 2004. "The Future of the Defence Firm in Small and Medium Countries." Defence and Peace Economics, 15:6, pp. 551-64.

Taylor, Travis. 2002. "Using Offsets in Procurement as an Economic Development Strategy." International Conference on Defence Offsets and Economic Development.

Udis, Bernard and Keith Maskus. 1991. "Offsets as Industrial Policy: Lessons from Aerospace." Defence Economics, 2, pp. 151-64. Trade.

United States Department of Commerce. Offsets in Defense

Versailles, David et Valérie Mérindol. 2006. Knowledge Transfers and R\&D Management: An Inquiry into The Problem of Transatlantic Complementarities. Defence and Peace Economics, 173, pp. 239-256.

Wessner, Charles ed. 1999. Trends and Challenges in Aerospace Offsets. Washington: National Academy Press.

World Economic Forum. 2008. The Global Competitiveness Report 2008-2009. 